\title{
Evaluation Of The Compound Muscle Action Potential In Diagnosis Of The Mild Carpal Tunnel Syndrome
}

\author{
Veysel Alcan *1 ${ }^{1}$ Murat Zinnuroğlu ${ }^{2}$, Gülçin Kaymak Karataş ${ }^{2}$, Mehmet Rahmi Canal ${ }^{3}$
}

\author{
Accepted 3rd September 2016
}

\begin{abstract}
Carpal tunnel syndrome (CTS) is the most common entrapment neuropathy. In most patients, the diagnosis can be proposed based on patient history and clinical symptoms, with electrophysical findings. The mild CTS may not produce any nerve conduction abnormalities and this can make standard conventional tests not enough in diagnosis the mild CTS. The aim of this study was to evaluate Compound Muscle Action Potential (CMAP) morphology as more sensitive and specific parameters without any additional testing for diagnosis the mild CTS. A total of seventy seven clinically diagnosed patients with CTS were prospectively enrolled. Data was evaluated from seventy normal hands and forty six hands with the diagnosis of the mild CTS with standard electrodiagnostic (EDX) tests and clinical findings. The specificity and sensitivity rate were calculated to evaluate the utility of CMAP negative peak (NP) morphology parameters evaluated duration (CMAP $\mathrm{NP}_{\text {Half-Duration }}$ and CMAP $\left.\mathrm{NP}_{\text {Full-Duration }}\right)$ and area $\left(\mathrm{CMAP} \mathrm{NP}_{\text {Half-Area }}\right.$ and CMAP NP $\left.\mathrm{Cull}_{\text {-Area }}\right)$ by comparing the standard EDX test (Median Distal Motor Latency (DML) and peak to peak amplitude of CMAP (CMAP NP Amplitude $_{2}$

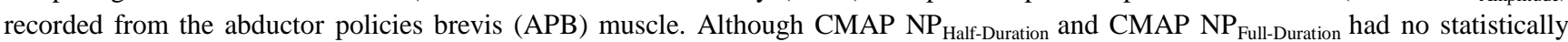
significantly difference between the mild CTS and normal group ( $p>0.05)$, DML, CMAP NP $\mathrm{Amplitude}_{\text {, }} \mathrm{CMAP}_{\mathrm{NP}} \mathrm{Half}_{\mathrm{Hrea}}$ and CMAP $\mathrm{NP}_{\text {Full-Area }}$ in the mild CTS group were statistically significantly different $(\mathrm{p}<0.05)$. The present study shown CMAP NP $\mathrm{Pull}_{\text {-Area }}$ had the highest sensitivity and moderate specificity rate $(90.0 \%$ and $42.2 \%$, respectively). Furthermore, it was confirmed again that DML was a valuable motor nerve conduction technique for the diagnosis of the mild CTS with high sensitivity and moderate specificity $(84.8 \%$ and $47.6 \%$, respectively), and it had more sensitive than CMAP $\mathrm{NP}_{\text {Half-Area }}$ high sensitivity and moderate specificity $(80.0 \%$ and $38.7 \%$, respectively). This study provided the evidence of CMAP NP $\mathrm{Full}_{\text {-Area }}$ and CMAP NP $\mathrm{Nalf}_{\text {-Area }}$ that could be predictors of the mild CTS
\end{abstract}

Keywords: carpal tunnel syndrome, motor nerve conduction study, clinical electromyography, signal processing.

\section{Introduction}

Carpal tunnel syndrome (CTS) is the most common entrapment neuropathy caused by median nerve compression at the wrist causing pain, paraesthesia, and functional problems [1]. Its prevalence ranges from $1-5 \%$ in the general population [2]. CTS is diagnosed on the basis of clinical signs and symptoms and confirmed by an electro diagnostic (EDX) tests. Nerve conduction study (NCS) and electromyography (EMG) are a standard part of the evaluation for CTS. They are useful to support the diagnosis of CTS, to assess severity, to provide a baseline for postoperative assessment in patients with unsatisfactory surgical result, and to exclude other abnormalities such as polyneuropathy, myopathy and radiculopathy [3]. The EDX tests rest upon the demonstration of impaired median nerve conduction across the carpal tunnel in the context of normal conduction elsewhere. Nerve compression results in damage to the myelin sheath and manifests as delayed distal latencies and slowed conduction velocities with resulting in a reduction of the median nerve compound motor action potential amplitude (CMAP) or sensory action potential amplitude (SNAP). Several EDX techniques have been established as standard and conventional methods for diagnose of CTS [4-5].

Although NCS and EDX tests have been widely used in diagnosing CTS, there are still ongoing debates particularly on

${ }^{\bar{l}}$ Dikmen Vocational High School, Ilker 1. Cad. , 06450, Dikmen, Ankara, Turkey

${ }^{2}$ Department of Physical Medicine and Rehabilitation, Gazi University

Faculty of Medicine, 06500, Ankara, Turkey

${ }^{3}$ Department of Computer Engineering, Gazi University Faculty of

Technology, 06500, Ankara, Turkey

* Corresponding Author: Email: alcanveysel@gmail.com accurate because sensitivities of EDX tests have ranged between $49 \%$ and $84 \%$ with specificities of $95 \%$ or higher. Furthermore, EDX test may have a substantial rate of false-negative findings, and so rely instead on clinical findings, especially in mild CTS that may not produce any nerve conduction abnormalities [6-9]. The patients have signs and symptoms of CTS, but the conventional studies may show equivocal or false negative. On the other hand, there are patients with median slowing at the wrists but who do not have CTS. Consequently, the main point of contradiction is that EDX tests yield negative results on symptomatic persons or positive results on no symptomatic persons [10]. This contradiction is more common in the diagnosis of the mild CTS.

Conventional wrist-to-abductor policies brevis (APB) motor conduction studies such as distal motor latency (DML) and amplitude of CMAP may not be sensitive enough to detect abnormalities (e.g., motor unit loss, conduction block), especially in mild CTS patients. It is noticeable that early and accurate diagnosis of CTS is essential for effective non-operative management and for valid identification of the surgical candidates [11]. Thus, more sensitive methods are needed and researchers tend to investigate other methods for verifying clinical and EDX findings with high sensitivity and specificity in the mild CTS [12].

It is generally accepted that median sensory conduction is more sensitive than motor conduction in the EDX of CTS. However, the low sensitivity of standard median motor EDX techniques in detecting motor axon abnormality also needs to be considered [13]. CMAP results from the sum of all the synchronously 
activated muscle fiber action potentials generated through all the axon destined to the APB muscle. The CMAP is an indirect measurement of the number of axons that conduct between the stimulating and recording points. A change in the CMAP emerges in peripheral nerve disorders. It is most prominent in entrapment neuropathies and demyelinating neuropathy, in which conduction is either blocked or delayed because of segmental demyelination [14]. Although distal CMAP duration prolongation is useful diagnostic tool for chronic inflammatory demyelinating polyneuropathies (CIDPs), the parameters analysed of the morphology of the distal CMAP can be also potentially useful parameters in the diagnosis of the mild CTS. In this manner, it cannot be needed significantly more time-consuming conventional EDX techniques which the patient can refuse extensive testing because of poor tolerance to electrical stimulation.

The aim of this study was evaluate diagnostic value of the halfduration and the full-duration of CMAP negative peak (NP) and the half-area and the full-area of CMAP NP as a complementary test in diagnosis of mild CTS. And then we compared this parameter's sensitivity and specificity with both DML and CMAP amplitude obtained conventional EDX tests.

\section{Methodology}

\subsection{Study Group}

A total seventy seven subjects (twenty five female, five male) with suspected CTS was retrospectively enrolled in Gazi University Faculty of Medicine between January 2014 and December 2014. After EDX tests in EMG Laboratory, seventy normal hands belonging to patients with suspected clinically CTS but no electrophysiological evidence of CTS and forty six symptomatic hands belonging to patients with mild CTS were included in the present study.

Physical examination consisted of evaluating of muscular strength, sensory function and provocative clinical test (Tinel signs) were performed before the EDX tests. The patients who have numbness, tingling pain, or paraesthesia in the median nerve distribution, and have precipitation of these symptoms by repetitive hand activities and relieved by resting, rubbing, and shaking the hand were included. The patients who have history or clinical signs suggesting systemic disease such as double-crush injury, diabetes, chronic renal failure, gout, rheumatoid arthritis, thyroid diseases and have clinical or electrophysiological signs suggesting pathological conditions such as myelopathy, polyneuropathy, radiculopathy, myopathy were excluded. We selected only the mild CTS diagnosed according to the criteria of our laboratory and used for diagnosis and classifying of the CTS $[15,16]$.

\subsection{Electrophysiological Measurements}

A Medelec Synergy EMG device (Medelec, Surrey,UK) was used in the study. Filters were set at $20 \mathrm{~Hz}$ and $2 \mathrm{kHz}$ for motor studies. Stimulation was supramaximal and sensitivity was 1 millisecond (ms). The sweep speed was set at $1 \mathrm{~ms} /$ division and sensitivity was selected as $20 \mu \mathrm{V} /$ division. Cup electrodes $(\mathrm{Ag}-\mathrm{Cl})$ were used for motor NCVs. In the NCVs, the distance between the recording electrodes is $3-5 \mathrm{~cm}$. The active electrode was over the endplate region of the main bulk of the APB muscle innervated by the nerve being tested in order to obtain the maximal response with no initial positivity. The reference electrode was over the tendon of the muscle. The ground electrode was placed on the between the stimulating and recording electrodes providing a zero voltage reference point. Electrical stimuli were delivered by a constant current stimulator through bipolar surface electrodes. The distance between two felts is $3 \mathrm{~cm}$ and the diameter of the electrodes is $8 \mathrm{~mm}$. It was applied supramaximal for a period of $0.1-0.5 \mathrm{~ms}$ and approximately and in a density of 10-30 mA. Skin temperatures were continuously monitored and maintained between $32-34^{\circ} \mathrm{C}$ during the procedures and the room temperature was maintained between at $22^{\circ} \mathrm{C}$ and $25^{\circ} \mathrm{C}$.

\subsubsection{Motor NCS}

DML was recorded with a distance of $5 \mathrm{~cm}$ between the stimulation point of the nerves at the wrist and the APB muscles. DML was measured from the stimulus onset to the initial negative response, and the CMAP amplitude was measured from baseline to negative peak and measured in millivolts $(\mathrm{mV})$, shown in Fig 1. Markers which indicated the onset, negative peak, positive peak and end of the CMAP waveforms were automatically determined by the Medelec Synergy software. In case of incorrectly placed, markers were manually repositioned.

\subsubsection{CMAP Morphology}

As shown in Fig.1, CMAP NP full-duration was measured from initial deflection from baseline (1) to the first baseline crossing (3). CMAP NP half-duration was measured from initial deflection from baseline (1) to the peak point on negative peak (2). The calculation of half-duration might be more accurate because fullduration of CMAP NP might return to baseline very slowly. In addition, CMAP NP half-area and CMAP NP full-area were calculated.

\subsubsection{Signal Analyses}

All CMAP duration and area measurements were manually marked and calculated by Signal5 Software (CED, micro1401, Camridge, UK). Using this software, CMAP signals were fullrectified and compared peak and baseline markers with not rectified original signal because a baseline shift or other electrical interference may lead to a problem for reliable measure of the area. The terminal positive deflection of the CMAP having multiple baseline crossings and phases was not calculated in the measurement because the end of the repolarization phase was frequently difficult and complex to determine $[17,18]$.

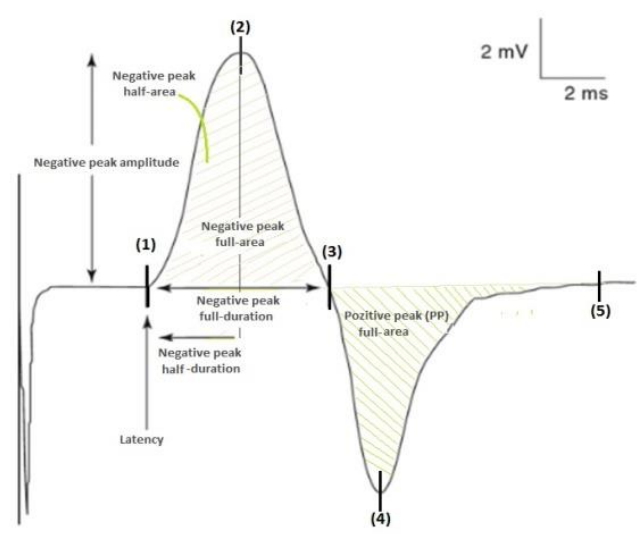

Figure 1. Components of the CMAP and signal analysis processes [17].

\subsection{Statistical Analyses}

The mean and standard deviations were calculated for statistical analysis using SPSS 20.1 (The Statistical Package for Social Sciences, Chicago, IL, USA). The two-tailed Student's t-test was used for comparative statistics. In order to assess the differences (age and gender) between the normal control group and the 
abnormal group, Chi-square test was performed. For the statistical significance, the probability level of $5 \%(\mathrm{p} \leq 0.005)$ was preferred.

Validity was assessed by the ability of CMAP amplitude, duration and area to discriminate between the mild CTS and the control groups using area under the receiver operator characteristics curve (ROC). The ROC curve was obtained by plotting the sensitivity of all possible cut-off points for a test on the $y$-axis against ' 1 -specificity' (false positivity) on the $\mathrm{x}$-axis. As a measure of the overall performance of a diagnostic test, the area under the ROC curve (AUC) was estimated using a form of the trapezoid method, and $95 \%$ confidence interval of the AUC was estimated by the bootstrap method approach. Accuracy was measured by the area under the ROC curve. One criterion for evaluating the cut-off point of a test was to maximize the sum of sensitivity and specificity. It was thought of optimally better cutoff for differentiating patients. It is known that searching for a more sensitive test might present the drawback of decreased specificity, leading to false positives [19]. It was considered cutoff points for both high sensitivity and high specificity.

\section{Results}

Totally seventy seven subjects with CTS suspicion were clinically examined with conventional EDX methods in EMG laboratory. While total twenty nine subjects (twenty five women
$(86.2 \%)$ and four men $(13.8 \%)$, age $=55.14 \pm 7.5)$ were diagnosed as the mild, total forty eight subjects (thirty nine women $(81.2 \%)$ and nine men $(18.8 \%)$, age $=41.62 \pm 13.8)$ were assessed with normal findings. Totally one hundred six teen hands (seventy normal hands and forty six asymptomatic hands) belong to subjects were included in this study. The characteristic of study population is given in Table 1.

Table 1. The characteristic of study group

\begin{tabular}{lccccc}
\hline \multirow{2}{*}{ Groups } & & \multicolumn{2}{c}{ Subjects } & \multicolumn{2}{c}{ Total } \\
\cline { 6 - 6 } & & N (\%) & $\begin{array}{c}\text { Age } \\
\text { Conean } \pm \text { SD) }\end{array}$ & N (\%) & $\begin{array}{c}\text { Age } \\
(\text { mean } \pm \text { SD) }\end{array}$ \\
\hline \multirow{2}{*}{ Montrol } & F & $39(81.2)$ & $40.8 \pm 12.6$ & & \\
& M & $9(18.8)$ & $45.1 \pm 18.8$ & $48(62.3)$ & $41.6 \pm 13.8$ \\
CTS & F & $25(86.2)$ & $55.5 \pm 7.7$ & & \\
\multirow{2}{*}{ Total } & M & $4(13.8)$ & $52.7 \pm 5.8$ & $29(37.7)$ & $55.1 \pm 7.5$ \\
& F & $64(83.1)$ & $46.5 \pm 13.1$ & $77(100)$ & $46.7 \pm 13.5$ \\
\hline
\end{tabular}

CTS $=$ carpal tunnel syndrome, $\mathrm{F}=$ female, $\mathrm{M}=$ male, $\mathrm{N}=$ number, $\mathrm{SD}=$ standard deviation

The comparison of the diagnostic competence of the parameters in terms of mean value, sensitivity, specificity and AUC are shown Table 2.

Table 2. Comparisons of parameters between the control and the mild CTS groups.

\begin{tabular}{lccccccc}
\hline \multicolumn{1}{c}{ Parameters } & $\begin{array}{c}\text { Control } \\
(\mathbf{m e a n} \pm \mathbf{S D})\end{array}$ & $\begin{array}{c}\text { Mild CTS } \\
(\mathbf{m e a n} \pm \mathbf{S D})\end{array}$ & $\mathbf{p}$ & $\begin{array}{c}\text { Cut-off } \\
\text { value }\end{array}$ & $\begin{array}{c}\text { Sensitivity } \\
(\boldsymbol{\%})\end{array}$ & $\begin{array}{c}\text { Specificity } \\
(\boldsymbol{\%})\end{array}$ & AUC \\
\hline $\mathrm{DML}(\mathrm{ms})$ & $2.91 \pm 0.3$ & $3.40 \pm 0.3$ & $\mathbf{0 . 0 0 0}$ & $\geq 6.8$ & 84.8 & 47.6 & 0.800 \\
$\mathrm{CMAP}_{\text {Amplitude }}(\mathrm{mV})$ & $14.66 \pm 3.4$ & $11.07 \pm 3.5$ & $\mathbf{0 . 0 0 0}$ & $\leq 11.0$ & 80.0 & 36.5 & 0.766 \\
$\mathrm{CMAP} \mathrm{NP}_{\text {Half_Duration }}(\mathrm{ms})$ & $4.9 \pm 0.0$ & $4.7 \pm 0.0$ & 0.331 & $\geq 4.5$ & 65.7 & 13.5 & 0.560 \\
$\mathrm{CMAP} \mathrm{NP}_{\text {Full_Duration }}(\mathrm{ms})$ & $10.5 \pm 0.00$ & $10.2 \pm 0.0$ & 0.208 & $\geq 9.8$ & 56.5 & 12.1 & 0.572 \\
$\mathrm{CMAP} \mathrm{NP}_{\text {Half_Area }}(\mathrm{mVms})$ & $26.5 \pm 0.0$ & $20.6 \pm 0.0$ & $\mathbf{0 . 0 0 0}$ & $\leq 20.5$ & 80.0 & 38.7 & 0.727 \\
$\mathrm{CMAP} \mathrm{NP}_{\text {Full_Area }}(\mathrm{mVms})$ & $60.8 \pm 0.0$ & $45.5 \pm 0.0$ & $\mathbf{0 . 0 0 0}$ & $\leq 41.9$ & 90.0 & 42.2 & 0.743 \\
\hline
\end{tabular}

$\mathrm{CTS}=$ carpal tunnel syndrome, $\mathrm{F}=$ female, $\mathrm{M}=$ male, $\mathrm{N}=$ number, $\mathrm{SD}=$ standard deviation

DML significantly differed between groups. There was statistically significant difference in terms of CMAP $\mathrm{NP}_{\text {Half-Area }}$

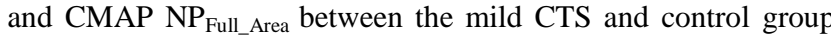
$(\mathrm{P}<0.001)$. However, there was no significant difference in terms of CMAP NP $\mathrm{Nalf}$ _Duration and CMAP NP $_{\text {Full_Duration between groups }}$ $(\mathrm{P}=0.331$ and $\mathrm{P}=0.208$, respectively).In the diagnosis of mild CTS, CMAP $\mathrm{NP}_{\text {Half_Area }}$ parameter (cut-off $\leq 41.9,90.0 \%$ sensitivity and $42.2 \%$ specificity) had the highest sensitivity and second high specificity rates. DML parameter (cut-off $\geq 6.82$, $84.8 \%$ sensitivity and $47.6 \%$ specificity) had the highest specificity and second high sensitivity rates. Although $\mathrm{CMAP}_{\text {Amplitude }}$ (cut-off $\leq 11.00,80.0 \%$ sensitivity and $36.5 \%$ specificity) and CMAP NP Half_Area (cut-off $\leq 20.5,80.0 \%$ sensitivity and $38.7 \%$ specificity) parameters showed similarly high sensitivity and specificity rates, Both of CMAP $\mathrm{NP}_{\text {Half_Duration }}$ and CMAP NP $\mathrm{Null}_{\text {_Duration }}$ parameters had similarly the lowest sensitivity and specificity rates.

AUC values of DML $(\mathrm{AUC}=0.800,95 \% \mathrm{CI}), \mathrm{CMAP}_{\text {Amplitude }}$
(AUC 0.766, $(95 \% \mathrm{CI}), \mathrm{CMAP} \mathrm{NP}_{\text {Half_Area }}(\mathrm{AUC}=0.727,95 \%$ $\mathrm{CI})$ and CMAP NP $\mathrm{Null}_{\text {FArea }}(\mathrm{AUC}=0.753,95 \% \mathrm{CI})$ parameters having a "good" ratio were significantly different from 0.5 and that therefore it was considered that these parameters had an ability to distinguish between the mild CTS and healthy subjects. However, it was found that both of CMAP $\mathrm{NP}_{\text {Half_Duration }}$ $(\mathrm{AUC}=0.560,95 \% \mathrm{CI})$ and CMAP $\mathrm{NP}_{\text {Full_Duration }}(\mathrm{AUC}=0.572$, $95 \% \mathrm{CI})$ parameters having a "fail" rate had no ability of the test to correctly classify those with and without the mild CTS. ROC curves belong to all parameters is shown in Figure 2.

\section{Discussion}

The major finding of this study there were significant differences in the all the CMAP amplitudes, DML, CMAP Amplitude $_{\text {N }} \mathrm{NP}_{\text {Half_Area }}$ and CMAP NP Full_Area $_{\text {between the mild CTS and normal control }}$ groups. 




(a)



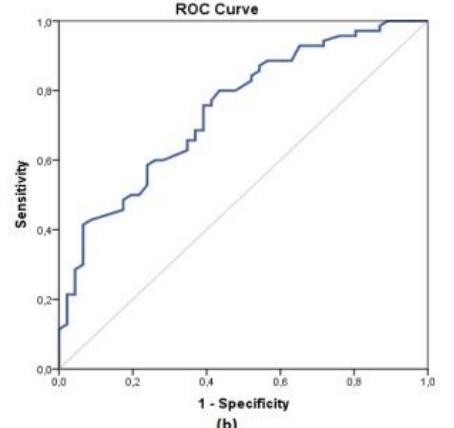

(b)

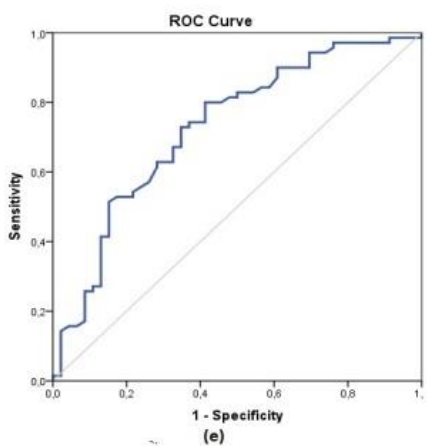

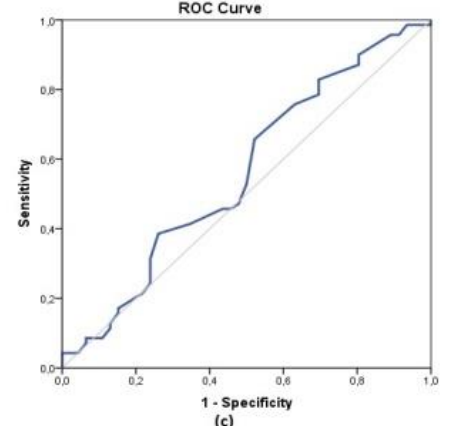

(c)

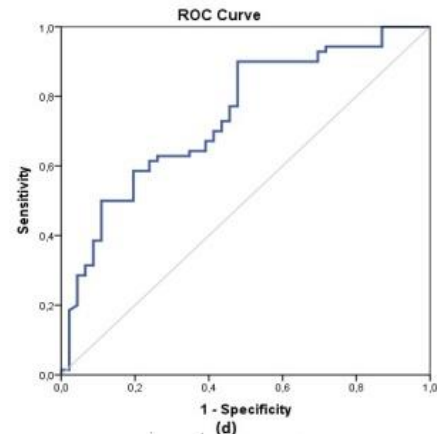

Figure 2. ROC curves (a) MDL (b) $\mathrm{CMAP}_{\text {Amplitude }}$ (c) CMAP NP $\mathrm{Palf}_{\text {_Duration }}$

(d) CMAP NP Full_Duration $_{\text {( }}$

(e) CMAP NP Half_Area $_{\text {, and (f) CMAP NP Full_Area }}$

This result on stimulating at the wrist may be a manifestation of axonal loss, conduction block or a combination. In the mild CTS, the primary abnormality observed with EDX measures was conduction slowing, which represents focal demyelination. Whereas distal latencies can reflect nerve conduction slowing of the fastest fibers, CMAP duration can depend on temporal dispersion among the distal motor fibers. Furthermore, some factors (the distribution of conduction velocities of individual axons within the nerve, the distance between stimulating and recording electrodes, the morphology of each single-fiber action potential and phase cancellation between individual single-fiber action potentials) might have influenced CMAP morphology and in particular CMAP duration.

Padua et al. claimed that $\mathrm{CMAP}_{\text {Amplitude was decreased only in }}$ patients with severe CTS as a result of the loss of motor units [15]. On contrary this, we found that $\mathrm{CMAP}_{\text {Amplitude }}$ was not decreased only in patients with severe CTS as a result of the loss of motor units but also in mild CTS. Unlikely our finding, Nashed et al. found that there was no difference in peak-to-peak MUAP amplitude between the mild CTS and the control groups [20]. It can be result in due to their very small study group (nine control group and eight mild CTS)

In many previous studies, CMAP NP full-duration prolongation assessed by measuring NP is commonly used in CIDP as diagnostic parameter [21-25]. CMAP duration prolongation can be rare in conditions that lead to distal latency prolongation in CTS. But we found that CMAP NP full-duration prolongation has not been incorporated in the diagnostic criteria for mild CTS. Similarly, Cleland et al. found that, in contrast to CIDP, dispersion of the median CMAP was uncommon, even in advanced carpal tunnel syndrome, being seen in only eight of one hundred three $(7.8 \%)$ hands [18]. Isose et al., in comparison of CMAP duration between normal controls and CTS patients, found very low sensitivity (for cut-off $=5.8 \mathrm{~ms} .23 \%$ sensitivity and for cut-off $=6.6 \mathrm{~ms}, 12 \%$ sensitivity) like our results [26].

The significant difference shown in NP full and half area may be considered as a manifestation of a very mild affection of motor axonal loss in even the mild CTS cases which are accepted as normal in terms of motor fibers involvement regarding the classification. Similar findings were found in DML which is also in normal range in mild cases. The classification suggests evaluating cases as moderate when the DML is abnormal (prolonged as compared with the normal limits).

\section{Limitations}

The main limitation of our study was the lack of sufficient and reliable cut-off values for CMAP duration, amplitude and area. Larger studies are needed to confirm the reference range of CMAP with diagnostic studies including larger sample sizes and well-defined study groups because cut-off values can be influenced by the choice of patients with axonal loss in the control group. Other limitation of our study is the control group who were referred to the ENMG laboratory with the suspicion of CTS. Some of these patients might be very mild cases in whom the EDX tests are normal. Therefore allocation of control subjects who are also clinically normal would be more valuable to detect milder differences.

\section{Conclusions}

In mild CTS, motor fibres can be more often affected than was originally thought and CMAP area also can reflect the number of muscle fibers in the CMAP. In this regard, we suggest distal CMAP area would be added into one of the EDX criteria for mild CTS in addition to conventional motor NCS. For further studies, it can be evaluated with new studies comprising large study groups and patient groups in different stages of CTS and including SNAP parameters (e.g., duration, area and amplitude) as well as wrist-elbow motor NCS including CMAP parameters (e.g., duration, area and amplitude). 


\section{References}

[1] Bradlley W.G. and Daroff R.B. Neurology in clinical practice, Carpal Tunnel Syndrome, 2nd ed., Boston, 2002, pp.1893-1894.

[2] Roquelaure Y., Ha C., Pelier-Cady M.C., et al. Work increases the incidence of carpal tunnel syndrome in the general population, Muscle Nerve, Vol. 37, 2008, pp. 477482.

[3] Preston D.C. and Shapiro B.E., Median neuropathy. In: Electromyography and neuromuscular disorders: Clinical electrophysiologic correlations, Butterworth Heinemann, Boston 231, 1998.

[4] Jablecki C.K., Andary M.T., Floeter M.K., et al. Second AAEM literature review of the usefulness of nerve conduction studies and needle electromyography for the evaluation of patients with carpal tunnel syndrome, Muscle Nerve, Vol.26, 2002, pp.S1-S53.

[5] Jablecki C.K., Andary M.T., Floeter M.K., et al. Practice parameter: Electrodiagnostic studies in carpal tunnel syndrome: report of the American Association of Electrodiagnostic Medicine, American Academy of Neurology, American Academy of Physical Medicine and Rehabilitation. Neurology, Vol.58, 2002b, pp.1589-1592.

[6] Jablecki C.K., Andry M.T., So Y.T., Wilkins D.E., Williams F.H. Literature review of the usefulness of nerve conduction studies and electromyography for the evaluation of patients with carpal tunnel syndrome, Muscle Nerve. Vol.16, 1993, pp.1392-1414.

[7] Rosenbaum R. Carpal tunnel syndrome and the myth of El Dorado, Muscle Nerve, Vol. 22, 1999, pp. 1165-1167.

[8] Keith MW, Masear V, Chung KC, Maupin K, Andary M, Amadio PC, et al. American Academy of Orthopaedic Surgeons Clinical Practice Guideline on diagnosis of carpal tunnel syndrome. J Bone Joint Surg Am, Vol. 91, 2009, pp.2478-2479.

[9] Kilmer DD, Davis BA. Electrodiagnosis in carpal tunnel syndrome. Hand Clin, Vol.18, 2002, pp.243-247

[10] Homan M.M., Franzblau A., Werner R.A., Albers J.W., Armstrong T.J., Bromberg M.B., Agreement between symptom surveys, physical examination procedures and electrodiagnostic findings for the carpal tunnel syndrome. Scand J Work Environ Health, Vol.25, 1999, pp.115-124.

[11] Szabo R.M., Slater R.R., Farver T.B., Stanton D.B., Sharman W.K. The value of diagnostic testing in carpal tunnel syndrome, J Hand Surg Am, Vol.24, 1999, pp.704714.

[12] Kohara N. Clinical and electrophysiological findings in carpal tunnel syndrome, Brain Nerve, Vol.59, 2007, pp. 1229-1238.

[13] Di Guglielmo G., Torrieri F., Repaci M., Uncini A. Conduction block and segmental velocities in carpal tunnel syndrome, Electroencephalogr Clin Neurophysiol, Vol.105, 1997, pp.321-327.

[14] Thaisetthawatkul P., Logigian E.L., Herrmann D.N., Dispersion of the distal compound muscle action potential as a diagnostic criterion for chronic inflammatory demyelinating polyneuropathy, Neurology Vol.59, 2002, pp.1526-1531.

[15] Padua L., LoMonaco M., Gregori B., Valente E.M., Padna R., Tonali P., Neurophysiological classification and sensitivity in 500 carpal tunnel syndrome hands," Acta Neurol Scand, Vol.96, 1997,.pp.2117.
[16] Baysal A., Kuruoğlu R., Beyazova M., et.al. Normal popülasyonda sinir iletimi değerleri,".Ege Nörolojik Bilimler Dergisi, Vol.6, 1989, pp.9-15.

[17] Katirji B., Kaminski H. J., Ruff R. L., Neuromuscular Disorders in Clinical Practice, 2nd ed., Newyork, USA, Springer, 2014.

[18] Cleland J.C., Logigian E.L., Thaisetthawatkul P., Herrmann D.N., Dispersion of the distal compound muscle action potential in chronic inflammatory demyelinating polyneuropathy and carpal tunnel syndrome, Muscle Nerve, Vol.28, 2003, pp.189-193.

[19] Singh, G. Determination of Cutoff Score for a Diagnostic Test, The Internet Journal of Laboratory Medicine, Vol.2, 2006, pp.1.

[20] Nashed J., Hamilton-Wright A., Stashuk D.W., Faris M., McLean L., Assessing motor deficits in compressive neuropathy using quantitative electromyography, Journal of Neuro Engineering and Rehabilitation, Vol.7, 2010, pp.39.

[21] Cleland J.C., Malik K., Thaisetthawatkul P., Herrmann D.N. and Logigian E. L., Acute Inflammatory Demyelinatıng Polyneuropathy: Contribution Of A Dispersed Distal Compound Muscle Action Potential to Electrodiagnosis, Muscle Nerve, Vol.33, 2006, pp.771-777.

[22] Rajaballya Y. A., Lagardeb J., Cassereauc J., Vialab K. Fournierb E. and Nicolas G., A European multicentre reappraisal of distal compound muscle action potential duration in chronic inflammatory demyelinating polyneuropathy, European Journal of Neurology, Vol.19, 2012, pp.638-642.

[23] Lewelt A.J., Krosschell K.J., Scott C., et al., Compound Muscle Action Potential and Motor Function in Children with Spinal Muscular Atrophy, Muscle Nerve. Vol. 42, Iss.5, 2010, pp.703-708.

[24] Lagarde J., Viala K., and E. Fournier. Is Total Duration Of Distal Compound Muscle Action Potential Better Than Negative Peak Duration In The Diagnosis Of Chronic Inflammatory Demyelinating Polyneuropathy?, Muscle Nerve, Vol.49, 2014, pp.895-899.

[25] Cleland J.C., Logigian E.L., Tharsetthawatkul P. and Herrmann D.N., Dispersion Of The Distal Compound Muscle Action Potential In Chronic Inflammatory Demyelinating Polyneuropathy And Carpal Tunnel Syndrome, Muscle Nerve, Vol.28, 2003, pp.189-193.

[26] Isose1 S., Kuwabara1 S., Kokubun N., et al., Utility of the distal compound muscle action potential duration for diagnosis of demyelinating neuropathies, Journal of the Peripheral Nervous System, Vol.14, 2009, pp.151-158. 